\title{
The Key Steps toward Automation of the Fixture Planning and Design
}

\author{
Attila Rétfalvi, Mihály Stampfer \\ Subotica Tech, Marka Oreškovića 16, 24000 Subotica, Serbia \\ ratosz@vts.su.ac.rs; stampfer@vts.su.ac.rs
}

Abstract: In automating fixture planning and design two major directions can be distinguished - automated dedicated fixture design, and automated modular fixture design. In this paper, the main steps that are needed for automated modular fixture planning and design are presented, and an integrated process planning and fixture design system developed following these steps - is introduced. These main steps are feature recognition, systematization of the fixturing subtasks, defining the fixturing feature determination rules, systematization of the modular elements, and systematization of the element selection rules.

Keywords: modular fixture design; CAPP

\section{Introduction}

Automated fixture design means a process, in which, without human interaction, an appropriate fixture is designed for a given workpiece. Since nowadays the 3D modeling software is very widespread, as input for a system that is capable of automated fixture design should serve the 3D model of the workpiece, complemented with technological data like tolerances, surface roughness, etc. In the past there were numerous attempts to develop such a system, but full automation is not provided by any of the systems developed so far. More or less human interaction is needed in defining input data, and the user also has the surveillance role - he evaluates the solution. In this way, faults due to unforeseeable errors can be avoided.

Since different things are important for the designer, and different things are important for the process engineer, the design features can differ from manufacturing features; so if one wants to automate fixture design, a program is needed which can - on the 3D model of the workpiece - automatically find all machining and fixturing features and extract their relevant data. The next step should be a systematic evaluation of the extracted data and restructuring the data into a format that matches the needs of the fixture design. The entities of which the workpiece geometry is constituted should be examined according to fixturing 
aspects such as feasibility for supporting, for positioning and/or for clamping. When appropriate base surfaces are found, the system should look for appropriate fixturing elements, place them at the proper place and in this way build a feasible fixture for the given workpiece.

The system that is presented in this paper uses a neutral file format (IGES [1]) as input, in order to ensure platform independence, and recognizes the most common technological (manufacturing and fixturing) features. Then the user defines which surfaces should be machined, with what precision, and with what surface roughness; the user also prescribes the dimension, shape and relationship tolerances. The program examines the features and surfaces of the workpiece and tries to find the best supporting, positioning and clamping solution, taking in consideration the prescribed tolerances. Finally, it selects concrete modular fixture elements out from the database, and puts these elements on adequate place, building this way a fixture.

\section{Literature Overview}

Since during fixture design there are frequently recurring tasks and since the number of possible solutions is huge, process engineers have long been using computers to make their work faster and easier. There have been numerous attempts to develop programs that will automate, or at least considerably speed up, the fixture design. Some work has focused on automated feature recognition, others have focused on fixture construction, and there were also some who tried to solve both tasks.

One of the first systems, which builds a fixture from modular elements for machining a given workpiece was made by A. Márkus et al [2]. The supporting, locating and clamping bases were interactively given by the process engineer, and the system tried to build an appropriate fixture from some pre-assembled element combinations. The system ensures that the solution must not be more complicated than an available feasible more simple solution, it ensures that fixture elements are over the palette, and it checks the space usage and the interference. The user has to accept the proposed solution; if he refuses it, the system tries to find another solution. Chep et al [3] describe a method for restructuring the data stored in a CAD model into a form that is appropriate for CAPP systems for the automatic definition of machining operation sequence. These generalized data in the objectoriented database can also be used for the fixture planning and design. Prabhu et al [4] report a system that from 2D drawings (saved in DXF or IGES format) automatically extracts different kind of features, the dimensions and their attributes. The program has a text parser to interpret the different notes on the drawings. Arivazhagan et al [5] have developed a feature recognition system, which uses syntactic pattern recognition technique. Their machinable volume identifier program recognizes different kind of slots, blind slots and steps. 
Subrahmanyam`s [6] heuristic-based volume decomposition method combines design feature usage (direct method) with a volume decomposition technique called heuristic slicing. In this way, the cell number (number of potential removal units) is halved compared with the cell-based approach, and the feature recognition time is decreased significantly. The fixturing algorithm in this research works only for single setup parts, and only flat surfaces are taken into consideration as potential fixturing surfaces. Kakish et al. in [7] outline a knowledge-based fixture design system. They focus on determining the design parameters and specifications of modular fixtures. Zhou et al [8] use the adjacent surface graph method in combination with feature tree reconstruction for feature recognition. This direct feature recognition system is able to find different kinds of slots, steps and holes. Rameshbabu et al [9] present a hybrid feature recognition method that combines volume subtraction and a face adjacency graph to cut recognition time. The feature identification is based on the number of feature faces and the adjacency count of each feature face. Their program recognizes different kinds of slots and steps. Wu et al [10] interpret the base principles of an algorithm that (with the assumption of having the primary positioning given) looks for the secondary and tertiary positioning points with the help of a linkage mechanism theory; the search for clamping points does a thorough investigation of IRC triangle and its same directed edges. The aim is to find a locating plan that ensures unambiguous, stable workpiece locating and where the loading and unloading is not hindered by the fixture elements. Boyle et al [11] studied the most recent works published about Computer Aided Fixture Design (CAFD), and they state that the CAFD is segmented in nature and that greater focus is needed on supporting detailed fixture design. They developed a case-based reasoning fixture design method. Xu et al [12] give an overview of the most commonly applied techniques in Computer Aided Process Planning, and they stress the need for integrated solving of the manufacturing related tasks and the importance of the environmentally conscious production. Alacron et al [13] developed a fixture planning and design system using the functional design theory. The user interactively prescribes the functional requirements, and the system, after defining the locating, supporting and clamping faces, selects modular elements and puts them in the defined place. Paris and Brissaud [14] present a process planning system that, after feature recognition assisted by an expert, associates machining processes to machining features and then organizes them into a global machining plan of the workpiece. Finally, it includes recommendations on fixturing features and determines the positioning quality, stability and cutter accessibility indices. Kumar et al [15] introduce an interactive fixture design system in which the user defines the fixturing surfaces and the program builds an interference free fixture. Perremans in [16] presents an expert system that in the possession of the fixturing features builds a modular fixture. In order to make the system manufacturer independent, he uses contact features, assembly features and tightening features to describe the modular elements. Vukelić et al [17] introduce an interactive combined (case-based and generative) system for drilling fixtures. Their system 
looks for an existing fixture solution in the database on the basis of the workpiece code; if does not find an appropriate existing solution, then it helps the user to generate a new fixture. Bansal et al [18] made an indirect feature recognition system that starts from the CAD model of the workpiece saved in STEP format. It determines the fixturing points by taking into consideration the prescribed tolerances, feature dependencies, manufacturing rules, fixture stability and ease of workpiece loading/unloading. In order to find the best fixturing points (which ensure the minimum tolerance deviances) the system slices the workpiece at different heights.

\section{Feature Recognition}

Feature recognition means the identification and extraction of the application relevant data from the part geometry [18]. Depending on the function, we distinguish design, manufacturing and fixturing features. The design features are groups of surfaces that are generated in a similar way; only some of their parameters differ - in this sense we can speak about protruded, swept, round, etc. features. Manufacturing features are those groups of surfaces that are made (usually removed) with the same tool or tool combination, with the same cutting parameters; they can also be those surface groups that serve as base surfaces during the assembly. In this sense machining features are different countersink and counterbore holes, slots, pockets, etc. Fixturing features are those surfaces or surface groups that are used for supporting, locating and/or clamping of the workpiece during the machining. The machining and fixturing features together are called technological features. As modeling software has evolved more and more commands for design feature generation have been built into them, but when one wants to use the features generated by them for process planning purposes, most often, he/she must first convert those into technological features. Since there are many different $3 \mathrm{D}$ modeling programs and since every program stores the data in a slightly different way, some neutral file formats (such as IGES, STEP, etc.) have been developed in order to ensure that a model made with one modeling program can be opened with other modeling programs, not only with the one with which it was made. When a model is stored in a neutral format, the design features are lost; only surface types (e.g. cylindrical, conical, etc.) are stored, so process planning - when it starts from a CAD model stored in neutral format - must begin with feature recognition.

The first step of feature recognition is the regeneration of the geometrical entities (points, curves, surfaces) that the model consists of and the classification and structuring of these data. The points are classified as start point, end point, midpoint, center point, point on curve, or point on surface. The curves consist of curve pieces, and these pieces are rated in three groups: straight line, conical sections and other, while curves are marked as inner or outer boundary curves. 
The surfaces can be planar, cylindrical, conical and other. The orientation of the surfaces is also important information: the orientation can be horizontal, vertical or angled. The convexity property is important as well. Of course the characteristic data of each curve piece (such as length, radii, etc.) and surface (such as normal, size, etc.) are also determined. After this classification comes the identification of common curves and curve pieces of the surfaces. In the next step, the neighboring surfaces of the surfaces with common conical section are checked for type, and this continues until a terminating surface (a full conic or a noncylindrical, non-conical surface that has not with actual surfaces coaxial cylindrical or conical surfaces) is reached. In this way surface groups are formed. Of course one has to leave out the found surface groups from further investigation in order that the same surface group not be found more times. Next, it is determined whether the found surface groups (potential features) form a feature and if yes, what kind of feature they form. Thus, we are looking for different kinds of holes (through or blind, with or without sinkage, with or without thread, with or without a slot for Saager-ring, etc.). Afterwards, different raised (boss) and sunken (pocket or slot) surfaces are looked for. Finally, it is determined if there are planar surfaces whose heights are the same, and could serve as supporting, locating or clamping surfaces. The feature recognition is introduced in more detail in [20].

\section{Prescribing Technological Data}

Technological data indicates those workpiece data that have influence on the kind of applicable machining process, on the tool dimensions and on the cutting parameters, and through these on the final fixture. Such data are, for example, the depth of a hole, the precision of the position of that hole, the diameter of the hole, the precision of the diameter, and the desired surface roughness. The geometrical data of each feature (such as the diameter, depth, position) are automatically extracted from the 3D model, but the precision of the position, the precision of the diameter, and the surface roughness are not stored when the model is stored in neutral format. So when all features are recognized, one must define which of them should be machined and with what precision; one must define the relationship tolerances too. One can take the recognized features one by one and prescribe the technological data. In the case of most features, the precision and the desired surface roughness must be prescribed. In the case of holes, in addition to the precision and surface roughness, one must define if it is threaded or not, and one must also define the initial state, i.e. whether it is pre-cast or drilled in full material. When there are more identical features (for example 5 counter sink holes with thread M6) the program will ask the user if all of them should be machined in the same way. Thus it is enough for the user to define her/his expectations one time: it is not necessary to do it five times. After that, the user selects the tolerance related features in pairs and prescribes the kind of relationship tolerance that binds 
the two features, as well as the precision of that tolerance. The prescribed precision of the relationship tolerances has a great impact on the structure and on the needed precision of the fixture.

\section{Defining the Fixturing Type, Surfaces and Points}

Since the system is developed for box-shaped parts, the first review is of the most common supporting, locating and clamping types used in the cases of box-shaped parts.

\subsection{Types of Supporting, Locating and Clamping [19]}

Box-shaped parts, especially gearbox casings, are most often machined on horizontal machining centers. Considering technological facilities of horizontal machining centers and analyzing existing clamping fixtures according to the position of the supporting surface of the workpiece, there are 3 types of supporting (Figure 1): (1) Horizontal (denoted with "pos1”), (2) Vertical (“pos2"), (3) Vertical with the ability of partial machining of the supporting face ("pos3").

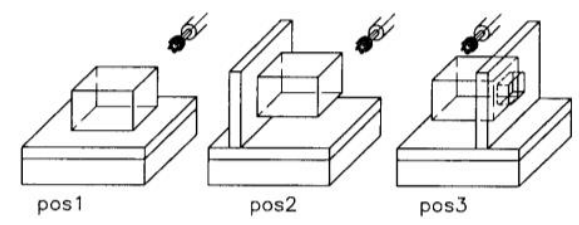

Figure 1

Supporting (Plane locating) types

There are 4 types of side locating (guiding) established (Figure 2): (1) side locating with the help of surfaces adjoining to the supporting face, (2) side locating with the use of two inside diameters on the supporting face, (3) side locating with the utilization of one inside diameter laying on the supporting face and one face adjacent to the supporting face, (4) side locating with the application of two threaded joints on the supporting face.

On the basis of the clamping force direction, one can distinguish between perpendicular clamping (s1), when the clamping force is perpendicular to the supporting surface, and parallel clamping (s2), when the clamping force is parallel with the supporting surface (Figure 3). 


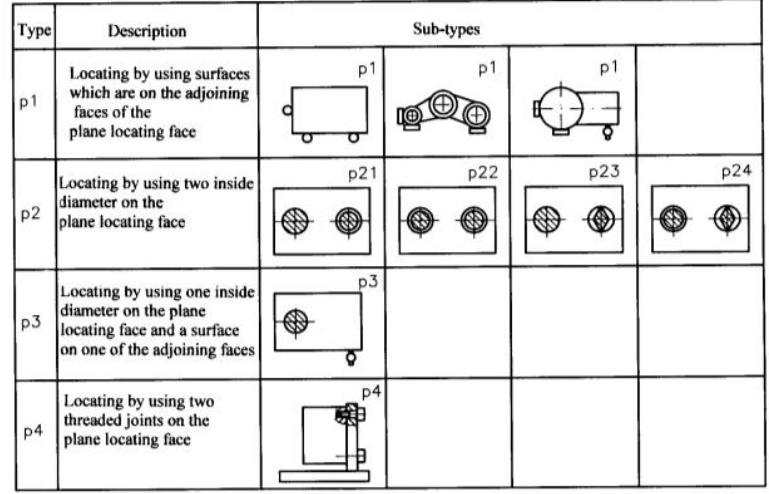

Figure 2

Types of side locating

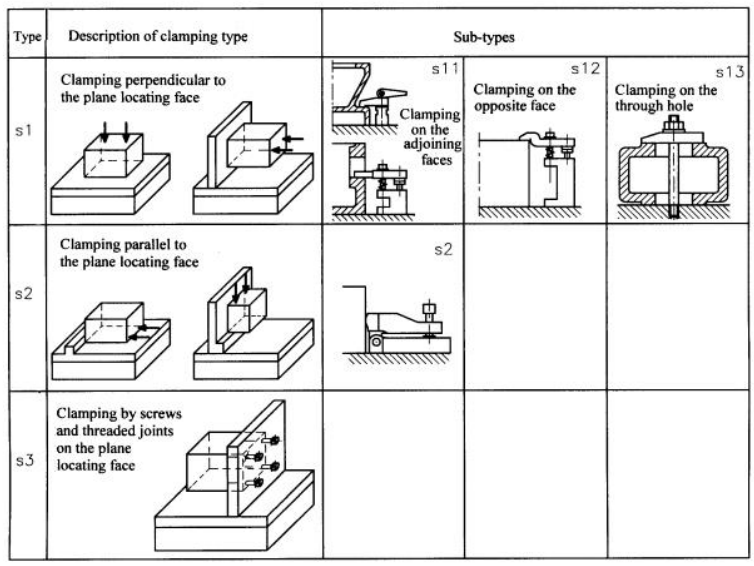

Figure 3

Types of clamping

The basic type s1, depending on the location of clamping faces, can be further divided into subtypes s11, s12 and s13. In the case of s11, the clamping surfaces are the closest parallel faces to the plane-locating (supporting) surface. In the case of $\mathrm{s} 12$, the clamping surface(s) is on the opposite side of the plane locating face. By s13 the clamping is carried out using a trough hole on the workpiece.

One special way of clamping is clamping by screws and joints on the plane locating face (s3). In this case, the clamping forces act perpendicular, but the force transmission happens in a different way.

The number of clamping points is also a very important characteristic of clamping. One distinguishes between clamping in one, two, three or four points. If the previous basic types are supplemented with this information, the results are the 
possible clamping types: s11_2, s11_3, s11_4; s12_2, s12_3, s12_4; s13_1, s13_2; s2_1, s2_2; s3_2, s3_3, s3_4. In the enumerated notation the last number denotes the number of clamping points.

\subsection{Suitable Surfaces for Locating and Clamping}

\subsubsection{Suitability for Plane Locating}

The suitability of a surface for plane locating depends on the shape and size of the surface. The best surfaces for plane locating are planar surfaces, then intermittent planar surfaces, surfaces on different parallel planes, cylindrical surfaces, and lastly a combination of cylindrical and planar surfaces.

The largest possible surface must be chosen for supporting the workpiece. The minimal allowed size of supporting surfaces is given as a percent of the largest dimensions of the part.

\subsubsection{Suitability for Side Locating}

a) Suitability for side locating type pl: Side locating can be divided into guiding and end supporting.

Suitability for guiding must be tested based on 3 aspects: shape, size and position of the surface.

- According to the shape of the guide locating element, the useable faces are: planar face, two planar faces, two cylindrical faces, a combination of cylindrical and planar surfaces, and single cylindrical surface.

- The typical dimension of a guiding surface must not be less than $35 \%$ of the longest dimension of the plane locating face.

- According to the position of the guiding element they must belong to adjoining faces of the plane locating face.

Suitability for end supporting must be tested based on two aspects: the shape and position of the surface.

- According to the shape of the surface, planar or cylindrical surfaces can be applied.

- According to the position, they must be on a face that is adjacent to both the locating and guiding faces.

b) Suitable surfaces for side locating type $p 2$

According to the shape of the surface, there should be two holes on the plane locating face. The distance between the holes must not be less than $35 \%$ of the greatest length of the plane locating face.

Suitable surfaces for other side locating types are defined in a similar way. 


\subsubsection{Suitability for Clamping}

The following principles must be respected during selection of clamping surfaces:

- The awaking forces should push the workpiece to the fixture.

- For the sake of rigid clamping, we must minimize the moments acting on the workpiece.

- The clamping should ensure positive rigidity.

- The clamping force must not deform the workpiece.

- The greatest shear force should be transmitted in a form-close way.

The clamping devices can be divided into two groups:

I. the clamping force changes with the deformation of the workpiece or the clamping device (screws, cams, wedges, springs, etc); or

II. the clamping force is constant (hydraulic, pneumatic, magnetic, etc.)

Of course the constant clamping force is better, but it can be achieved only with more expensive devices. So if the variation of clamping force is not too significant, one prefers elements from the first group.

Exactly which device will be used depends on the shape, size and location of the surfaces eligible for clamping.

For s1.1, the eligible surfaces are flat surfaces, through holes, or pockets; for s1.2 flat surfaces, intermittent planar faces or cylindrical surfaces; for s1.3 flat ring-like surfaces, flat frame-like surfaces or group of cylindrical surfaces (with axes perpendicular to the supporting face); for s2 flat surfaces, or intermittent planar faces; and for $\mathrm{s} 3$ threaded holes.

The location of clamping surfaces is on the opposite side to the supporting face at s1.1, s1.2 and s1.3; and at s1.3, the center of the hole is approximately coincident with the center of the supporting surface. At $s 2$ there are flat faces opposite to the locating side, and they are near the supporting surface. At s3 there are threaded holes on the supporting surface and the distance between them is great enough (bigger than $40 \%$ of the height).

The size of the clamping face(s) should be large enough to ensure the settlement of clamping devices and to ensure appropriate clamping pressure. At s3, M6 or greater thread is needed.

In addition to these, one must check whether any of the above mentioned principles are violated. 


\subsection{Determination of the Clamping Points}

For smaller parts, it is enough to clamp the part in two points, but a larger workpiece should be clamped in at least three points, and if there is enough space, it would even be advisable to do the clamping at four points. In order to distribute the clamping forces uniformly, the clamping area is divided into sectors and zones (Figure 4).

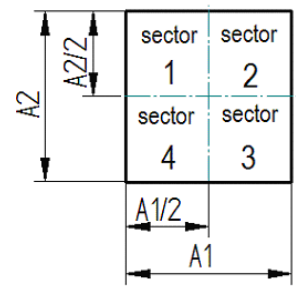

\begin{tabular}{|c|c|}
\hline \multicolumn{2}{|c|}{$\begin{array}{l}\text { The position of the clamping points at clamping } \\
\text { type } \mathbf{s 1 1}, \mathbf{s 1 2} \text { and } \mathbf{s 3}\end{array}$} \\
\hline $\begin{array}{l}\text { Clamping } \\
\text { in two } \\
\text { points }\end{array}$ & $\begin{array}{l}\text { - } \text { sectors } 1,3 \\
\text { - } \text { sectors } 2,4 \\
\text { - } \text { central zones } 1-4,2-3 \\
\text { - } \text { central zones 1-2, 3-4 } \\
\end{array}$ \\
\hline $\begin{array}{l}\text { Clamping } \\
\text { in three } \\
\text { points }\end{array}$ & $\begin{array}{l}\text { - } \text { sectors 1,2 and central zones 3-4 } \\
\text { - } \text { sectors 1,4 and central zones 2-3 } \\
\text { - } \text { sectors 3,4 and central zones 1-2 } \\
\text { - } \text { sectors 2,3 and central zones 1-4 }\end{array}$ \\
\hline $\begin{array}{l}\text { Clamping } \\
\text { in four } \\
\text { points }\end{array}$ & $\begin{array}{l}\text { - } \text { sectors } 1,2,3,4 \\
\text { - } \text { central zones } 1-2,2-3,3-4,1-4\end{array}$ \\
\hline
\end{tabular}

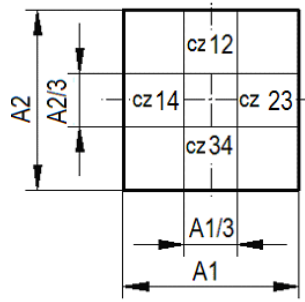

Figure 4

The division of the clamping area into sectors and zones

The layout of the clamping points for different cases can be seen in the table near the pictures. When determining the position of the clamping points, one must take care about the following: the clamping points must be over supporting points or as close to them as possible, and they must not be over features with precise tolerances.

\section{Fixture Configuration}

Fixture configuration means the selection of concrete elements for different fixturing tasks and the determination of their exact position and orientation.

The first step of fixture design is the systematization of the modular elements. Due to the high number of modular elements, the most commonly used elements for locating and clamping of box-shaped parts were chosen from the Kipp catalogue [21]. This reduces the search space and with it the searching time. 


\subsection{Systematization of the Modular Elements}

The elements of a modular fixture can be divided into three groups:

a) base elements

b) functional elements

c) adapting elements

Base elements establish contact between the machine tool table and the fixture. This group includes different palettes, grid plates, and angle grid plates. (Table 1)

Functional elements are those elements that come in contact with the workpiece to fulfill a concrete function such as supporting, locating or clamping. In this group belong supports (Table 2), locators (Table 3), clamps (Table 5), and multifunctional elements (Table 4).

These groups are subdivided according to similarity of function into further subgroups, and a typical representative element of each subgroup is introduced in the tables. Every subgroup receives an ID in order to facilitate the referencing. These IDs are used in the next subsection (0), where the selection rules are systematically presented.

In order to reduce the number of needed elements, fixture manufacturers combine functions and thus offer multifunctional elements, too (Table 4). Of course, the smaller the number of the elements in a fixture, the more stable and precise it is.

Adapting elements (Table 6) are not always used. Sometimes the clamping surface is too high, or due to the shape of the workpiece, the supporting surface must be elevated or the locating elements lengthened. In such cases, one uses adapting elements to bridge the distance between the functional and the base elements.

The modular elements in the database are stored under coded names of the Kipp catalogue, where the first four plus three numbers refer to the type and subtype of the modular element. The following two numbers denote the size of the joining surfaces, while the last three or four numbers refer to some of the more relevant parameters of the element such as width, length or height. For example, in the code 8000081123240 , the 8000 signifies that it is a grid plate with holes on every $50 \mathrm{~mm} .081$ indicates there are holes for socket head screws to facilitate fixing of the plate onto angle plate elements. 12 means the grid holes are M12 holes. 32 and 40 mean the width of the plate is $320 \mathrm{~mm}$ and length is $400 \mathrm{~mm}$. 
Table 1

Base plates

\begin{tabular}{|c|c|c|}
\hline ID & Element & Name \\
\hline EB1 & & $\begin{array}{c}\text { Grid } \\
\text { Plates }\end{array}$ \\
\hline EB2 & & $\begin{array}{l}\text { Angle } \\
\text { grid } \\
\text { plates }\end{array}$ \\
\hline
\end{tabular}

Table 2

Supporting elements

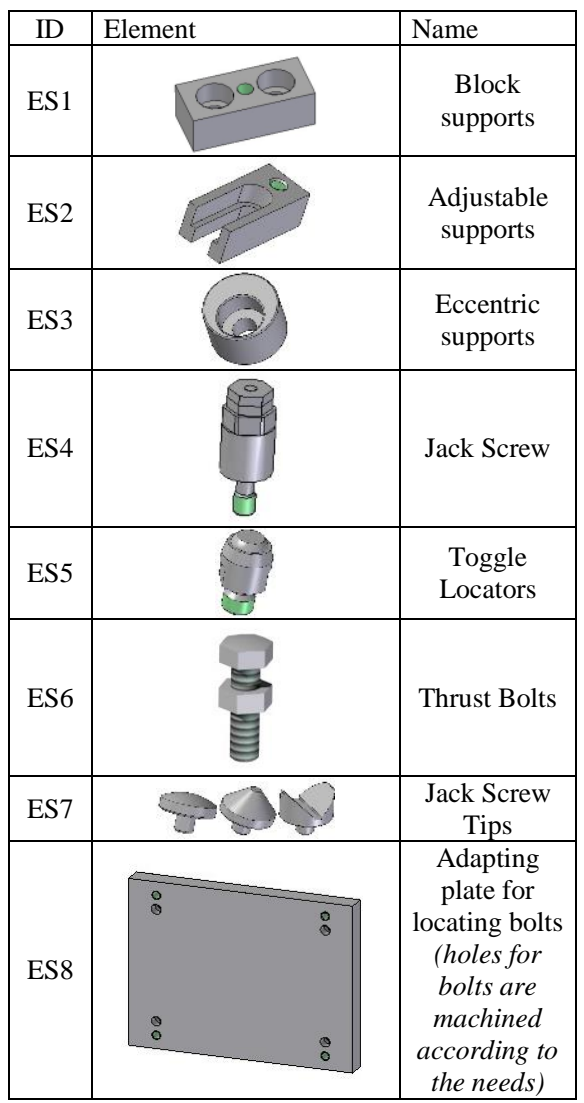

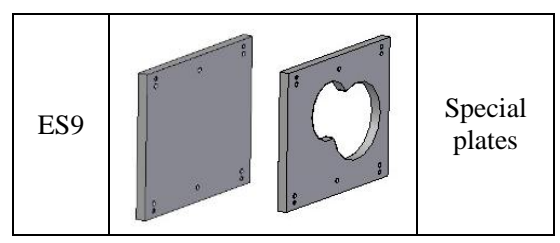

Table 3

Locator elements

\begin{tabular}{|c|c|c|}
\hline ID & Element & Name \\
\hline EP1 & & $\begin{array}{c}\text { Locating } \\
\text { supports }\end{array}$ \\
\hline EP2 & & \\
\hline EP3 & & \\
\hline EP4 & & \\
\hline EP5 & & \\
\hline
\end{tabular}

Table 4

Multifunctional elements

\begin{tabular}{|c|c|c|}
\hline ID & Element & Name \\
\hline EM1 & & $\begin{array}{c}\text { Adjustable } \\
\text { support with a } \\
\text { step }\end{array}$ \\
\hline EM2 & & Point Clamps \\
\hline EM3 & & $\begin{array}{c}\text { Adjustable Point } \\
\text { Clamp }\end{array}$ \\
\hline
\end{tabular}


Table 5

Clamping elements

\begin{tabular}{|c|c|c|}
\hline ID & Element & Name \\
\hline EC1 & & $\begin{array}{c}\text { Pin-End } \\
\text { Strap }\end{array}$ \\
\hline EC2 & & $\begin{array}{c}\text { Goose- } \\
\text { Neck } \\
\text { Strap }\end{array}$ \\
\hline EC3 & & \\
\hline EC4 & & \\
\hline EC5 & & \\
\hline EC7 & & $\begin{array}{c}\text { Hook } \\
\text { Clamps }\end{array}$ \\
\hline & & \\
\hline
\end{tabular}

Table 6

Adapting elements

\begin{tabular}{|c|c|c|}
\hline ID & Element & Name \\
\hline EA1 & & $\begin{array}{c}\text { Pin-End } \\
\text { Strap }\end{array}$ \\
\hline EA2 & & \\
\hline EA3 & & \\
\hline
\end{tabular}

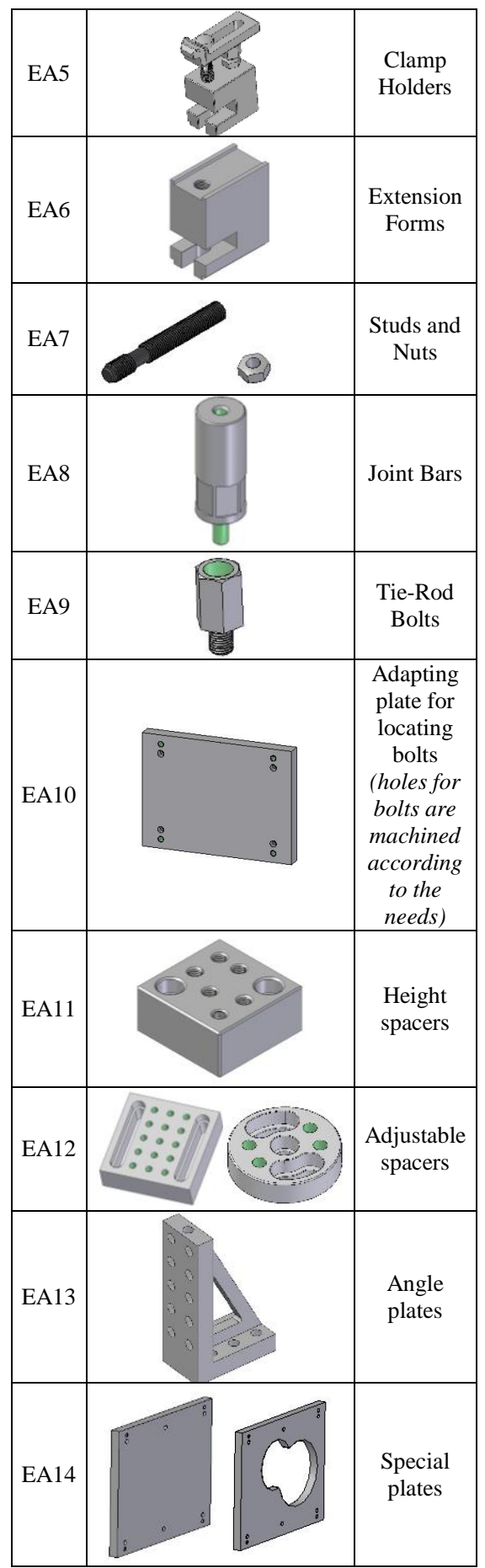




\subsection{Systematic Presentation of the Selection Rules of Modular Elements}

First the base element is selected in accordance with the size of the machine table and the type of the supporting (plane locating). Next the locators (and if needed, the adapting elements) are selected. Finally, the clamping (and if needed, adapting) elements are selected.

The selection rules are systematically ordered in matrices, and the columns contain the tasks. In the rows there are the elements and the conditions under which the elements can be used. $\mathbf{0}$ in the matrices means the element in question cannot be used for the task in question; 1 means that it can always be used; other symbols indicate under what circumstances it can be used. The nomenclature used is:

- - NOT, $\quad \mathrm{V}-\mathrm{OR}, \quad \Lambda$ - AND, $\quad$ PL - planar surface,

CYL - cylindrical surface, $\quad$ GPC - group of one planar and one cylindrical surface.

\subsubsection{Selection of Base Elements}

The selection of base elements depends on the dimensions of the machine tool's table, the dimensions of the workpiece, the type of supporting, the type of clamping and the type of locating. If the machine tool is defined, the base element's selection matrix looks as follows:

Table 7

Selection rules of base elements

\begin{tabular}{|c|c|c|c|c|}
\hline \multirow{2}{*}{\multicolumn{2}{|c|}{ Base elements }} & \multicolumn{3}{|c|}{ Supporting type } \\
\cline { 2 - 5 } & pos1 & pos2 & pos3 \\
\hline 1 & EB1 & 1 & $\mathrm{~s} 3 \vee \mathrm{p} 4$ & 1 \\
\hline 2 & EB2 & 0 & $7 \mathrm{p} 4 \wedge \mathrm{s} 3$ & 0 \\
\hline
\end{tabular}

The presented system first selects the type of the base element in the function of the proposed types of supporting, locating and clamping (Table 7) and then selects the appropriate size. Since the machine tool was established, only the workpiece size is taken into consideration. For example, if the (by SUPFIX module) proposed supporting type is pos2 (Fig. 2), the positioning type is p3 (Fig. 3), and the clamping type is s13 (Fig. 4), then the angle grid plate (EB2) is selected. However, if the supporting type is pos3, then a simple grid plate (EB1) together with angle plates (EA13) and special plates (EA14) should be used, in order to ensure tool approachability to those strictly connected surfaces of the workpiece, which lie on the supporting side of the workpiece. In the next step, the program verifies the dimensions of the workpiece and establishes the width and length of the base element. The main criterion is that at least one free row of grid holes must stay on each side after the workpiece is positioned on the center of the plate. This criterion ensures that there will be enough places for locating and clamping elements. 
Grid plates and MC plates (EB1) can always be used for supporting type pos1 and pos3. For pos2, only when the clamping method is $\mathrm{s} 3$ or if the positioning type is p4. Angled grid plates (EB2) are used only for supporting type pos2, since they ensure greater rigidity, but can be used only when the positioning method is not $\mathrm{p} 4$ and clamping method is not $\mathrm{s} 3$.

The main elements of the code for selection of the base element are as follows:

base_element(ST,LT,CT,BE,) procedure $(i, i, i, o)$.

selectSize $(B E T, S U T, W, L)$ procedure $(i, o, o, o)$.

sizel $(w p D 1, w p D 2, S U T, W, L)$ procedure $(i, i, o, o, o)$.

(ST - supporting type, LT - locating type, CT - clamping type, BE -base element, BET - base element type, SUT - sub type, W - width, L-length, wpD1 - the width of the workpiece measured parallel to supporting surface, wpD2 - the length of the workpiece measured parallel to the supporting surface)

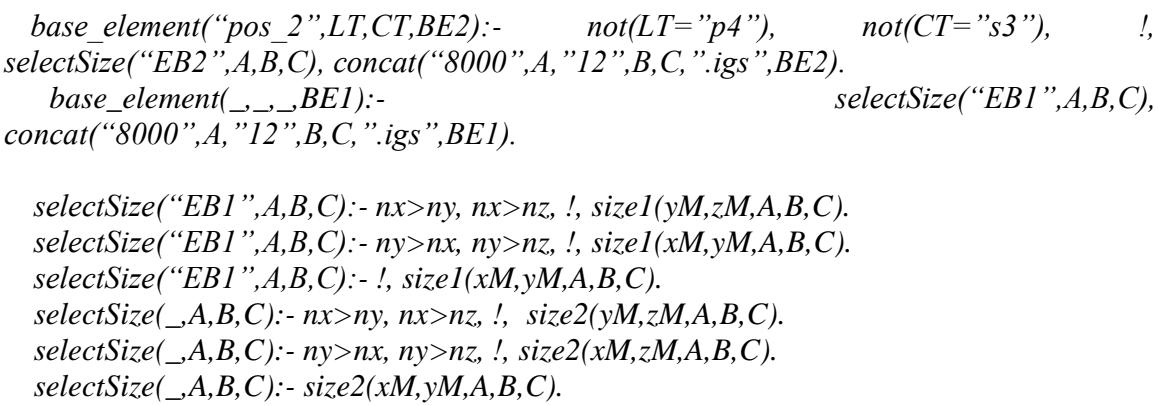

The program first checks if the proposed supporting type is pos_2: if yes, then it verifies if the locating type is $\mathrm{p} 4$, and if not it verifies if the clamping type is $\mathrm{s} 3$; if not, then it selects the angled grid plate (EB2). In any other case, the grid plate (EB1) is selected. The concrete base element is selected in the function of the workpiece dimensions that are parallel with the supporting surface. For example, if the supporting surface's normal vector points in $\mathrm{x}$ direction, then yMax and zMmax dimensions are considered. These data are obtained from the workpiece model during feature recognition (IPPO module) and are stored in appropriate variables.

\subsubsection{Selection of Locating Elements}

The locating task can be divided into two sub tasks, guiding and end stopping.

\subsubsection{Selection of Guiding Elements}

The selection of guiding elements first of all depends on the type of locating (Table 8). At types p2, p3 and p4 the subgroup for guiding elements is unambiguously determined, while at p1 the type of clamping and the type of the surface selected for guiding must also be taken into consideration. 
For example, if one wants to solve p1 type locating, and the clamping type is not s2 and there is an eligible plane surface for locating, then it is possible to use two locating supports (EP1), or two adjustable stops (EP2), or one locating support and one adjustable stop. But if the only possible clamping method is $\mathrm{s} 2$, then some kind of side stops (EP5) should be used.

Table 8

Selection of guiding locators

\begin{tabular}{|c|c|c|c|c|c|}
\hline \multirow{2}{*}{} & \multicolumn{4}{|c|}{ Type of locating } \\
\cline { 2 - 6 } & Locators & p1 & p2 & p3 & p4 \\
\hline 1 & $\mathbf{2}$ x EP1 & $7 \mathrm{~s} 2 \wedge \mathrm{PL}$ & 0 & 0 & 0 \\
\hline 2 & $\mathbf{2} \times$ EP2 & $7 \mathrm{~s} 2 \wedge \mathrm{PL}$ & 0 & 0 & 0 \\
\hline 3 & EP1 + EP2 & $7 \mathrm{~s} 2 \wedge \mathrm{PL}$ & 0 & 0 & 0 \\
\hline 4 & EP3 & 0 & 1 & 1 & 0 \\
\hline 5 & EP5 & s2 & 0 & 0 & 0 \\
\hline 6 & 2 x EP5 & s2 & 0 & 0 & 0 \\
\hline 7 & $\mathbf{2}$ x EP6 & CYL & 0 & 0 & 0 \\
\hline 8 & EP6 + EP2 & GC & 0 & 0 & 0 \\
\hline 9 & EP7 & 0 & 0 & 0 & 1 \\
\hline
\end{tabular}

Table 9

Selection of end stop locators

\begin{tabular}{|c|c|c|c|c|c|c|}
\hline \multirow{2}{*}{\multicolumn{2}{|c|}{ Locators }} & \multicolumn{5}{|c|}{ Type of locating } \\
\cline { 2 - 7 } & p1 & $\begin{array}{c}\text { p21 } \\
\text { p22 }\end{array}$ & $\begin{array}{l}\text { p23 } \\
\text { p24 }\end{array}$ & p3 & p4 \\
\hline 1 & EP1 & PL & 0 & 0 & 0 & 0 \\
\hline 2 & EP2 & PL & 0 & 0 & 1 & 0 \\
\hline 3 & EP3 & 0 & 1 & 0 & 0 & 0 \\
\hline 4 & EP4 & 0 & 0 & 1 & 0 & 0 \\
\hline 5 & EP5 & CYL & 0 & 0 & 0 & 0 \\
\hline 6 & EP6 & 0 & 0 & 0 & 0 & 1 \\
\hline
\end{tabular}

\subsubsection{Selection of End Stops}

The selection of end stops depends on the type of locating and also on the type of the selected end stop surface (Table 9).

\subsubsection{Selection of Supporting Elements}

The selection rules for supporting elements are shown in Table 10. It can be seen that in the majority of cases there is more than one acceptable solution. For example, if the supporting surface is a machined flat surface then different kinds of supports with flat supporting surfaces, or even grinded plates, can be used. What will be the determining factor is which of them fits to the workpiece dimensions best. When the supporting surface is larger, then block supports are preferred, while in the case of smaller supporting surfaces, adjustable or eccentric supports are the preferable choice. If the supporting surface is not machined yet, then toggle locators and thrust bolts or jack screws with tips must be used. So the system investigates the proposed type of supporting, locating and clamping, the number of proposed clamping points, and the size of the proposed supporting surface, and finally, it checks if the proposed supporting surface is machined.

\subsubsection{Selection of Clamping Elements}

Clamps are selected on the basis of the necessary type of clamping and on the basis of the type of the surfaces used for clamping (Table 11). The size of the clamping elements depends on clamping force needed and also on the vicinity of grid holes to the clamping place. 
Table 10

Selection rules of supports

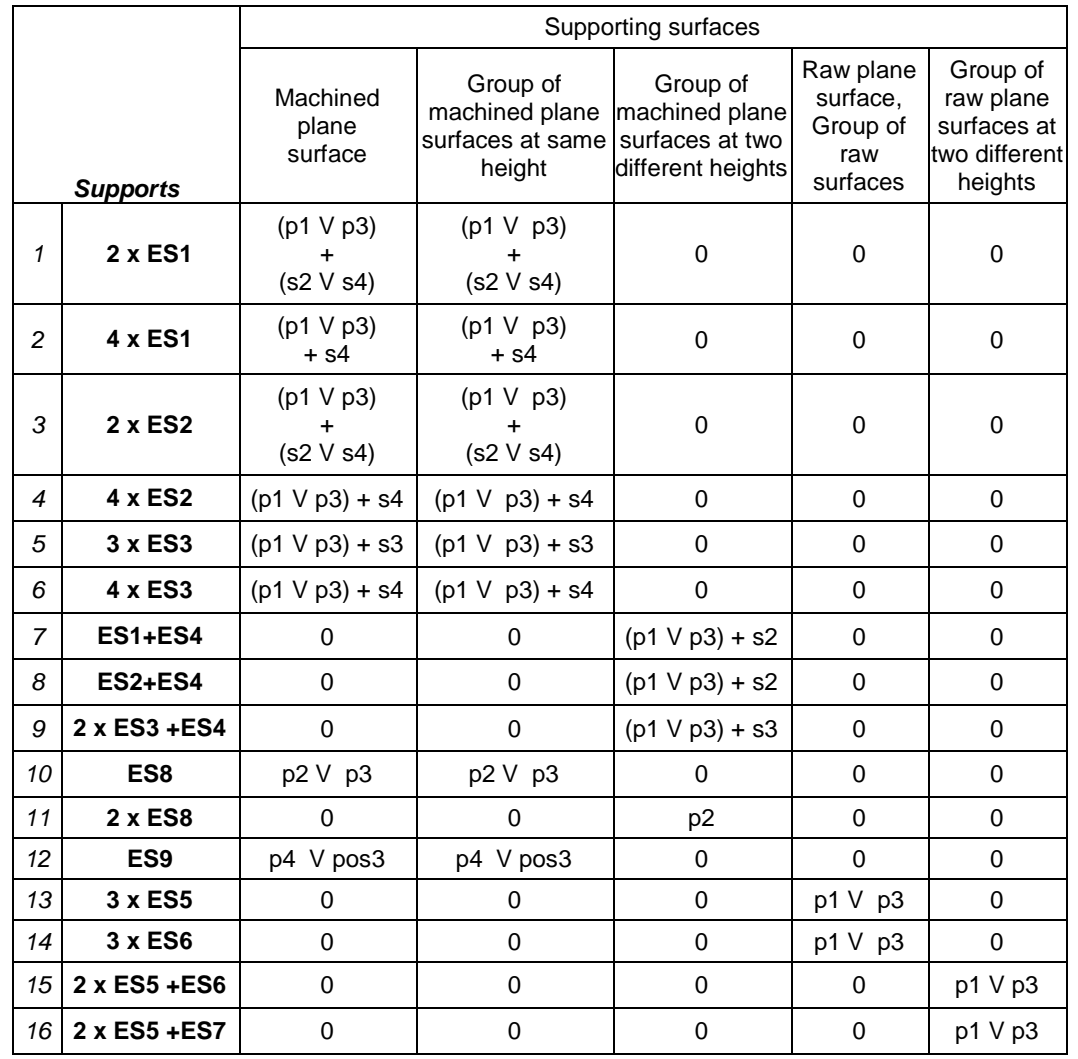

* here the numbers beside the letter $\mathbf{s}$ denote the number of clamping points

The number of clamps is equal to the number of necessary clamping points that are prescribed (by SUPFIX) in the conceptual solution of the fixture. It may happen that in different points different types of clamps are needed. Toggle locators (EC6) and thrust bolts (EC7) are used to increase the clamping range of hook clamps (EC3) at s12 type clamping. The usage of hook clamps is restricted by the grid hole positions: that is, they can be used if there is a grid hole (G HOLE) close enough to the proposed clamping point. If one wants to clamp the workpiece on an inner cylindrical surface, a pin-end strap (EC1) is to be used.

\subsubsection{Selection of Adapting Elements}

Adapting elements are configured so that they can be connected not only to the base and functional elements, but also to each other. Table 12 shows how the adapting elements can be combined with each other. Table 13 shows which functional element and adapting element can be combined. Theoretically the number of adapting elements used in a chain that connects a base and a functional 
element can be infinite, but of course the goal is to reduce the number of adapting elements to as few as is possible. The selected functional elements determine the type of applicable adapting elements, and the main task is to find a dimension combination that ensures the minimal number of chain elements.

Table 11

Selection of clamps

\begin{tabular}{|c|c|c|c|c|c|c|}
\hline \multirow{2}{*}{ Clamps } & \multicolumn{5}{|c|}{ Type of clamping } \\
\cline { 2 - 7 } & $\mathbf{s 1 1}$ & $\mathbf{s 1 2}$ & $\mathbf{s 1 3}$ & $\mathbf{s 2}$ & $\mathbf{s 3}$ \\
\hline 1 & EC1 & HOLE & 1 & 0 & 0 & 0 \\
\hline 2 & EC2 & 0 & 1 & 0 & 0 & 0 \\
\hline 3 & EC3 & G HOLE & G HOLE & 0 & 0 & 0 \\
\hline 4 & EC4 & 0 & 0 & 1 & 0 & 0 \\
\hline 5 & EC5 & 0 & 0 & 0 & 1 & 0 \\
\hline 6 & EC6 & 0 & 1 & 0 & 0 & 0 \\
\hline 7 & EC7 & 0 & 1 & 0 & 0 & 0 \\
\hline 8 & EC8 & 0 & 0 & 0 & 0 & 1 \\
\hline
\end{tabular}

Table 12

Mateability of adapting elements with other adapting elements and with base elements

\begin{tabular}{|c|c|c|c|c|c|c|c|c|c|c|c|c|c|c|c|c|}
\hline \multirow{2}{*}{$\begin{array}{l}\text { Adop } \\
\text { Elem }\end{array}$} & \multicolumn{16}{|c|}{ Adapting and Base Elements } \\
\hline & & & & & 5 & & & & & $\begin{array}{c}A \\
10\end{array}$ & $\begin{array}{c}\text { A } \\
11\end{array}$ & $\begin{array}{c}A \\
12\end{array}$ & $\begin{array}{c}A \\
13\end{array}$ & $\begin{array}{c}\text { A } \\
14\end{array}$ & $\begin{array}{c}E B \\
1\end{array}$ & $\begin{array}{c}E B \\
2\end{array}$ \\
\hline$A 1$ & 0 & 0 & 0 & 0 & 1 & 0 & 0 & 0 & ) & 0 & 0 & 0 & 0 & 0 & 0 & 0 \\
\hline A2 & 0 & 0 & 0 & 0 & 1 & 0 & 0 & 0 & & 0 & & 1 & 0 & 0 & 1 & 1 \\
\hline A3 & 0 & 1 & 0 & 0 & 0 & 0 & 0 & 0 & & 0 & 1 & 1 & 0 & 0 & 1 & 1 \\
\hline A4 & 0 & 1 & 1 & 1 & 0 & 0 & 0 & 1 ( & & 0 & 1 & 1 & 0 & 0 & 1 & 1 \\
\hline$A 5$ & 0 & 0 & 0 & 0 & 0 & 1 & 0 & 0 & & 0 & 0 & 0 & 0 & 0 & 1 & 1 \\
\hline A6 & 0 & 0 & 0 & 0 & 0 & 1 & 0 & 0 & & 0 & 0 & 0 & 0 & 0 & 1 & 1 \\
\hline$A 7$ & 0 & 1 & 0 & 0 & 0 & 0 & 0 & 1 & & 0 & 0 & 0 & 0 & 0 & 1 & 1 \\
\hline$A 8$ & 0 & 1 & 0 & 0 & 0 & 0 & 0 & 1 & & 0 & 1 & 1 & 0 & 0 & 1 & 1 \\
\hline$A 9$ & 1 & 1 & 1 & 1 & 0 & 0 & 0 & 0 & & 0 & 1 & 1 & 0 & 0 & 1 & 1 \\
\hline$A 10$ & 0 & 0 & 0 & 0 & 0 & 0 & 0 & 0 & & 0 & 0 & 1 & 0 & 0 & 1 & 1 \\
\hline$A 11$ & 0 & 0 & 0 & 0 & 0 & 0 & 0 & 0 & & 0 & I & 1 & 0 & 0 & 1 & 1 \\
\hline$A 12$ & 0 & 0 & 0 & 0 & 0 & 0 & 0 & 0 & & 0 & 0 & 0 & 0 & 0 & 1 & 1 \\
\hline$A 13$ & 0 & 0 & $v$ & 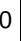 & 0 & v & 0 & 0 & & 0 & 0 & 0 & 0 & 0 & 1 & 1 \\
\hline$A 14$ & 0 & 0 & 0 & 0 & 0 & 0 & 0 & 0 & & 0 & 0 & 0 & 1 & 0 & 1 & 0 \\
\hline
\end{tabular}

Table 13

Mate ability of Functional elements with Base and Adapting elements

\begin{tabular}{|c|c|c|c|c|c|c|c|c|c|c|c|c|c|c|c|}
\hline \multirow[b]{2}{*}{$\begin{array}{l}\text { Func. } \\
\text { Elem. }\end{array}$} & \multicolumn{15}{|c|}{ Adapting and } \\
\hline & & & & & & & & & & & & & & & \\
\hline ES1 & ( & 0 & $\begin{array}{lll}0 & 0\end{array}$ & 0 & & & 0 & & & & & & & & \\
\hline ES2 & & 1 C & 0 & 0 & & & & & & & & & & & \\
\hline ES3 & & 1 . & $1 \mid 0$ & 0 & J & 10 & & & & & & & & & \\
\hline ES4 & & 1 . & 10 & 0 & & U & & & & & & & & & \\
\hline ES5 & & 1 & 10 & 0 & & & & & & & & & & & 1 \\
\hline ES6 & & 1 . & 10 & 0 & 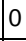 & jo & & & & & & & & & I \\
\hline ES7 & & 0 & \begin{tabular}{l|l}
0 & 1
\end{tabular} & $1 \mid 0$ & J & 0 & 0 & & & & & & & & 0 \\
\hline ES8 & & 0 & $0 \mid 0$ & 0 & U & 0 & 0 & & 0 & & & & & & 1 \\
\hline ES9 & & 0 & $\begin{array}{lll}0 & 0\end{array}$ & 0 & 10 & 10 & 0 & U & & & & & & & 0 \\
\hline$E P 1$ & & 1 . & 10 & 0 & & & & & & & & & & & 1 \\
\hline$E P 2$ & & 1 & 10 & 0 & U & & & & & & & & & & 1 \\
\hline EP3 & & 0 & 0 & 0 & 10 & 0 & 0 & 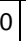 & 1 & & & & & & 0 \\
\hline EP4 & & 0 & 0 & 0 & 0 & 0 & 0 & 0 & & & & & & 0 & 0 \\
\hline EP5 & & 0 & 0 & 0 & & 0 & 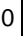 & & & & & & & & 1 \\
\hline EP6 & 0 & 0 & 0 & 00 & $\sigma$ & 0 & 0 & & & & & & & & I \\
\hline EP7 & 0 & 0 & 0 & 0 & 0 & 0 & 0 & U & 0 & & U & & & 0 & 0 \\
\hline$E C 1$ & & 0 & 0 & 11 & & 10 & & & & & & & & & 0 \\
\hline EC2 & 0 & 0 & 0 C & \begin{tabular}{l|l}
0 & 1
\end{tabular} & 0 & & & & 0 & & & & & U & 0 \\
\hline EC3 & & 1 & 0 & 0 & & & & & & 1 & 1 & 0 & & 1 & 1 \\
\hline$E C 4$ & ] & 0 & 0 & 0 & 0 & 1 & & 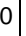 & & V & 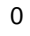 & 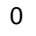 & & 0 & 0 \\
\hline EC5 & 0 & 0 & 0 & 0 & & & & & 0 & 1 & 1 & & & 1 & 1 \\
\hline EC6 & 1 & 0 & 0 & 0 & & & & & & 0 & 0 & & & 0 & 0 \\
\hline$E C 7$ & 1 & 0 & 0 & 00 & 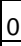 & & & U & 0 & 0 & 0 & 0 & 0 & 0 & 0 \\
\hline$E C 8$ & 0 & 0 & 0 & 0 & 0 & & & & 0 & & & & & & 0 \\
\hline
\end{tabular}


When a functional element is selected, the system instantly checks if there is a need for an adapting element. From the characteristic dimension (usually the height) of the surface with which the functional element is in contact is subtracted the position of the surface of the ground element, in the direction of the surfaces normal. The difference is reduced with the distance between the contact surfaces toward the workpiece and toward the adapter element. This should be bridged with an adapting element or elements. Naturally, the range of the adjustability of the functional element is taken into consideration.

\section{Test Example}

In Figure 5 a gearbox casing and its most important dimensions and the prescribed tolerances are presented. Figure 6 shows the proposals of the SUPFIX module on the first setup, and the proposed solution is: lay the workpiece on the violet ringlike surface, locate it with the help of the pink inner cylindrical surface and the gray rectangular surface, and finally clamp it over the green ring-like surface.
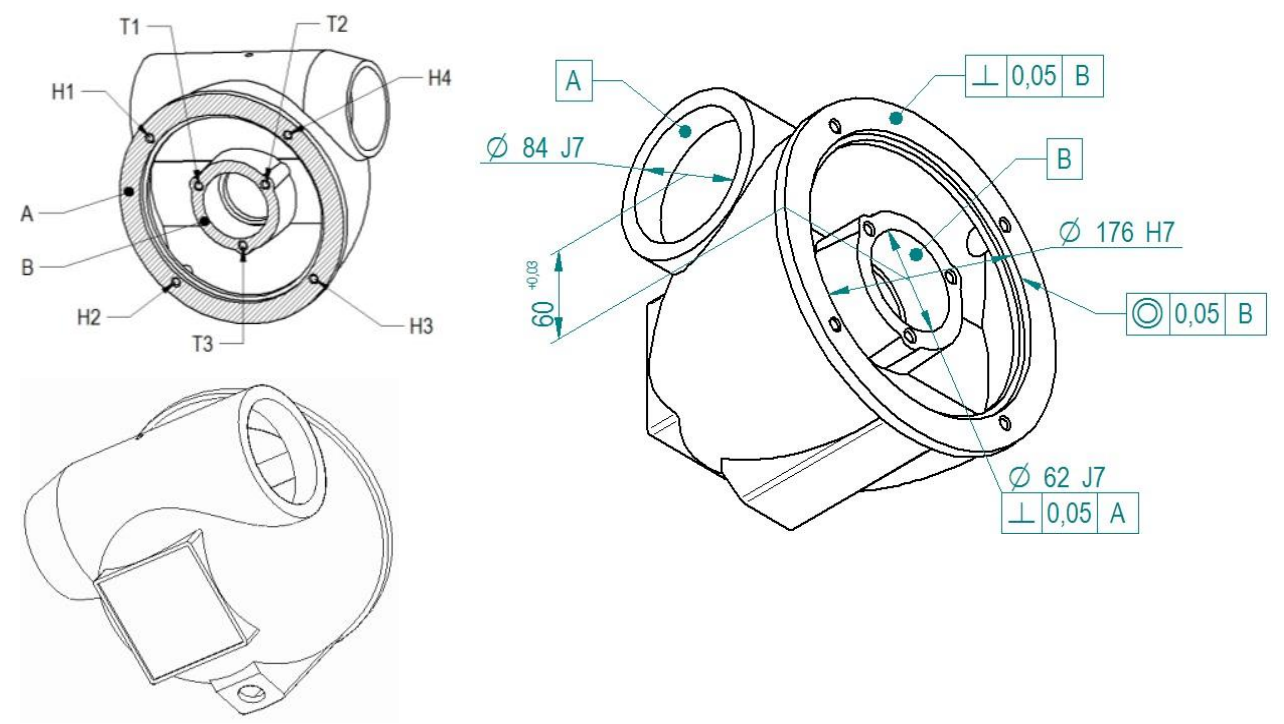

Figure 5

The workpiece, and the most important dimensions and tolerances

The proposed supporting type is "pos_1", the side locating is "p3", and the clamping type is "s_13". In the first (auxiliary) setup, two ring-like surfaces (A,B) and four $(\mathrm{H} 1, \mathrm{H} 2, \mathrm{H} 3, \mathrm{H} 4)$ plus three $(\mathrm{T} 1, \mathrm{~T} 2, \mathrm{~T} 3) \mathrm{M} 10$ threaded holes are machined. The (by FIXCO module) proposed fixture for this first setup and the Solid Edge assembly module (made by GLUE module) of the proposed fixture with and without the workpiece are also presented. The proposal for the second (main) 
setup in Figure 7 can be seen. During the second setup the workpiece should be laid on the violet ring-like surface, located with two threaded holes (green), and clamped over the four threaded holes (green). The proposed supporting is "pos_3", locating is "p4", and the clamping is "s3". The main elements of the fixture without the workpiece and the whole fixture with the workpiece are shown.
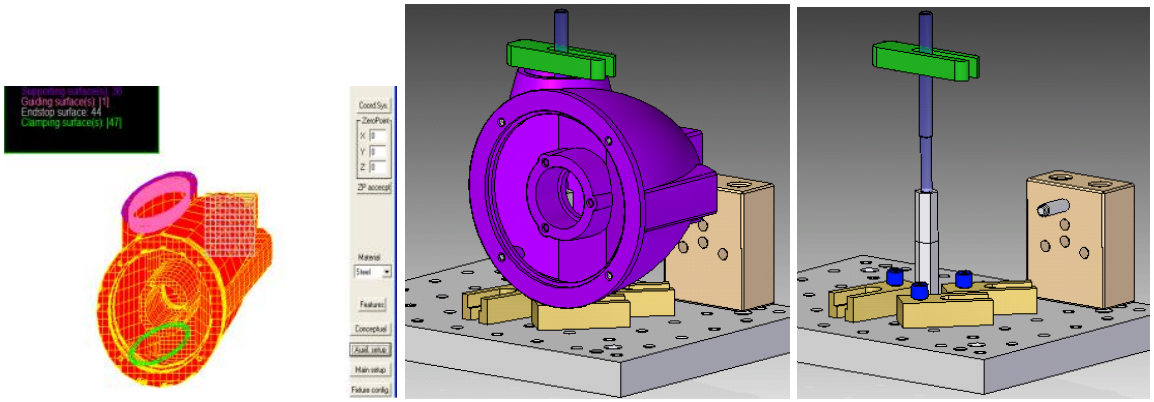

Figure 6

The proposed fixturing surfaces for the auxiliary setup, and the proposed fixture for the auxiliary setup
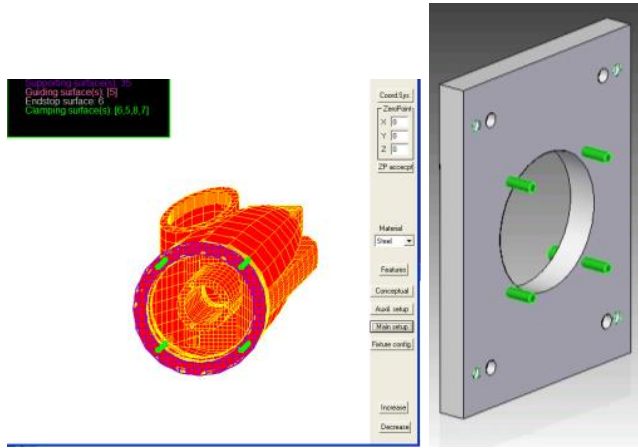

Figure 7

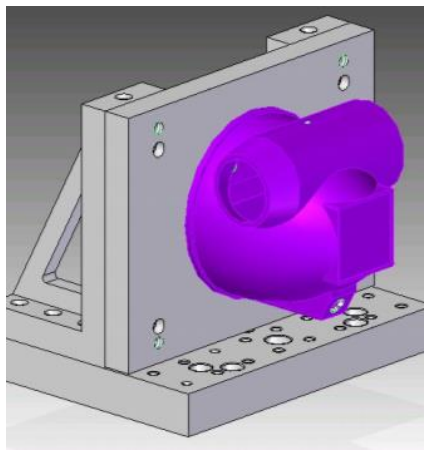

The proposed fixturing surfaces for the main setup, the adapter plate with clamping screws and the proposed fixture for the main setup

\section{References}

[1] US PRO (2008): Initial Graphics Exchange Specifications 5.3

[2] A. Márkus, Z. Márkusz, J. Farkas és J. Filemon (1984): Fixture Design using Prolog: An Expert System, Robotics and Computer Integrated Manufacturing 1 (2): 167-172

[3] A. Chep, L. Tricarico, P. Bourdet, L. Galantucci (1998): Design of Objectoriented Database for the Definition of Machining Operation Sequences of 3D Workpieces, Computers in Industrial Engineering 34 (2): 257-279 
[4] B. S. Prabhu, S. Biswas, S. S. Pande (2001): Intelligent System for Extraction of Product Data from CADD Models, Computers in Industry 44:79-95

[5] A. Arivazhagan, N. K. Mehta, P. K. Jain (2009): A STEP AP 203 - 214based Machinable Volume Identifier for Identifying the Finish-Cut Machinable Volumes from Rough Machined Parts, Int. J. Adv. Manuf. Technol. 42:850-872, doi 10.1007/s00170-008-1659-2

[6] S. R. Subrahmanyam (2002): A Method for Generation of Machining and Fixturing Features from Design Features, Computers in Industry 47: 269287

[7] J. Kakish, P. L. Zhang, I. Zheid (2000): Towards the Design and Development of a Knowledge-based Universal Modular Jigs and Fixture Systems, J. of Intelligent Manufacturing 11:381-401

[8] X. Zhou, Y. Qiu, G. Hua, H. Wang, X. Ruan (2007): A Feasible Approach to the Integration of CAD and CAPP, Computer Aided Design 39:324-338

[9] V. Rameshbabu, M. S. Shunmugam (2009): Hybrid Feature Recognition Method for Setup Planning from STEP AP 203, Robotics and Compurter Integrated Manufacturing 25: 393-408

[10] Y. Wu, S. Gao, Z. Chen (2008): Automated Modular Fixture Planning Based on Linkage Mechanism Theory, Robotics and Computer Integrated Manufacturing 24: 38-49

[11] I. Boyle, Y. Rong, D. C. Brown (2011): A Review and Analysis of Current Computer-Aide Fixture Design Approaches, Robotics and Computer Integrated Manufacturing 27:1-12

[12] X. Xu, L. Wang, S. T. Newman (2011): Computer-aided Process Planning A Critical Review of Recent Developments and Future Trends, International Journal of Computer Integrated Manufacturing 24 (1): $1-31$

[13] R. H. Alacron, J. R. Chueco, J. M. P. Garcia, A. V. Idiope (2010): Fixture Knowledge Model Development and Implementation Based on a Functional Design Approach, Robotics and Computer Integrated Manufacturing 26:56-66

[14] H. Paris, D. Brissaud (2005): Process Planning Strategy Based on Fixturing Indicator Evaluation, Int J Adv Manuf Technol 25: 913-922

[15] A. S. Kumar, J. Y. H. Fuh, T. S. Kow (2000): An Automated Design and Assembly of Interference Free Modular Fixture Setup, Computer-aided Design 32:583-596

[16] P. Perremans (1996): Feature-based Description of Modular Fixturing Elements: the Key to an Expert System for the Automatic Design of the Physical Fixture, Advances in Engineering Software 25: 19-27 
[17] Đ. Vukelić, U. Zuperl, J. Hodolic (2009): Complex System for Fixture Selection, Modification and Design, Int J Adv Manuf Technol 45:731-748

[18] S. Bansal, S. Nagarajan, N. V. Reddy (2008): An Integrated Fixture Planning System for Minimum Tolerances, Int J Adv Manuf Technol 38:501-513

[19] M. Stampfer (2009): Automated Setup and Fixture Planning System for Box-shaped Parts, Int J Adv Manuf Technol 45:540-552

[20] Rétfalvi A. (2011): IGES-based CAD Model Post Processing Module of a Setup and Fixture Planning System for Box-shaped Parts, IEEE $9^{\text {th }}$ International Symposium on Intelligent Systems and Informatics, September 8-10, 2011, Subotica, Serbia

[21] Heinrich Kipp Werk: Workholding Systems, Catalogue of the Modular Fixture Elements 